\title{
Change in Depression Symptomatology and Cognitive Function in Twins: A 10-Year Follow-Up Study
}

\author{
Inge Petersen, ${ }^{1}$ Matt McGue, ${ }^{1,2}$ Qihua Tan, ${ }^{1,3}$ Kaare Christensen, ${ }^{1,3,4}$ and Lene Christiansen ${ }^{1}$ \\ ${ }^{1}$ The Danish Twin Registry, Unit of Epidemiology, Biostatistics and Biodemography, Institute of Public Health, University \\ of Southern Denmark, Odense, Denmark \\ ${ }^{2}$ Department of Psychology, University of Minnesota, Minneapolis, Minnesota, USA \\ ${ }^{3}$ Department of Clinical Genetics, Odense University Hospital, Odense, Denmark \\ ${ }^{4}$ Department of Clinical Biochemistry and Pharmacology, Odense University Hospital, Odense, Denmark
}

\begin{abstract}
A complex interrelation exists between change in depression symptomatology and cognitive decline. Studies indicate either that depression is a direct risk factor for cognitive change over time, or vice versa. Longitudinal twin studies provide the possibility to unravel cause and effect of correlated traits. Here, we have applied twin modeling approaches to shed light on the genetic correlation between both level and change of depression symptomatology and cognitive functioning, and to further explore the bidirectionality of any such correlation using assessments of both phenotypes at two occasions 10 years apart. The study included 2,866 Danish twins with a mean age of 56.8 years at intake (range: 45-68 years). Of these, 1,267 were intact pairs. A total number of 1,582 twins (55\%), of whom 557 were intact pairs, participated in the follow-up survey. We found stable cross-sectional heritability estimates of approximately $60 \%$ for general cognitive abilities and $30 \%$ for affective depressive symptoms. There was a considerable decline in the mean cognitive performance over 10 years, whereas the mean affective depression symptoms score was stable and with no genetic contribution to any individual change. Additionally, we saw a small but significant cross-trait correlation at both occasions ( -0.11 and -0.09 , respectively), but cross-trait cross-occasion analysis revealed no evidence that either of the two traits predicts the other over a 10-year interval. Thus, our study was not able to detect any causal association between change in depressive symptomatology and cognitive decline in middle-aged and elderly people over a 10-year interval.
\end{abstract}

Keywords: depression, cognition, heritability, longitudinal, causation

Aging is accompanied by a decline in cognitive functioning, with the potential of progressing into dementia. Such changes may have significant consequences for the quality of life, and given the almost worldwide growth of the elderly, identification of possible modifiable risk factors for cognitive deficits is of central importance (Barnes \& Yaffe, 2011).

It is generally acknowledged that there is a large degree of comorbid correlation between depression symptoms (characterized by either clinically diagnosed depression or a high score on measures of depressive symptoms) and cognitive impairment (based either on a dementia diagnosis or as deficits on a battery of cognitive tests). There are several possible, but not necessarily mutually exclusive, explanations for this comorbidity. These include: (1) depressed mood can directly and independently increase the risk of dementia, (2) depression lowers the threshold for manifesting dementia, (3) depressive symptoms are a prodromal sign of evolving cognitive impairment, (4) depression is a consequence of a decline in cognitive functioning, or (5) the two conditions share common risk factors or pathological paths (Bennett \& Thomas, 2014). One potential source of shared etiology is a partly common genetic basis, that is, genetic factors that influence the susceptibility to experiencing depression symptoms may also have a direct effect on cognitive level or decline.

RECEIVED 6 November 2015; ACCEPTED 15 December 2015. First published online 16 February 2016.

ADDRESS FOR CORRESPONDENCE: Inge Petersen, The Danish Twin Registry, Unit of Epidemiology, Biostatistics and Biodemography, Institute of Public Health, University of Southern Denmark, J.B.Winsløws Vej 9B, 5000 Odense C, Denmark. E-mail: ipetersen@health.sdu.dk 
Although the temporal relationship between depression symptoms and cognitive deficits may still be debated (Huang et al., 2011; Jajodia \& Borders, 2011; Vinkers et al., 2004), a number of recent reviews and meta-analyses conclude that depressive symptoms can predict both mild cognitive decline and development of dementia over time (Bennett \& Thomas, 2014; Byers \& Yaffe, 2011; da Silva et al., 2013; Diniz et al., 2013; Gao et al., 2013). Of note though, many of the longitudinal studies included in these metaanalyses are unidirectional, only testing the a priori hypothesis of depression as a risk factor for dementia or cognitive decline and not the reverse. However, several recent papers have explored the directional nature of the correlation. The English Longitudinal Study of Ageing, a six-year followup survey with four assessment waves, used findings from 8,611 participants age 50 through 90 years and reported that higher levels of depression symptoms at baseline were associated with a slightly faster decline in cognitive functioning. Although this was only significant in individuals aged from 60 to 80 years, a similar non-significant tendency was seen in those aged from 50 to 60 years old. Contrary to this, depression status was reasonably stable over the years of follow-up, and cognitive status was not predictive of changes in depression symptoms (Gale et al., 2012). Using a cross-domain latent change analysis set-up in a 13year follow-up study of 2,299 participants 55+ years of age, the Longitudinal Aging Study Amsterdam demonstrated that baseline depressive symptoms were a robust predictor of cognitive decline. In contrast, only processing speed, not general cognitive ability, was related to an increase in depressive symptoms over time (van den Kommer et al., 2013). Similarly, using an autoregressive latent trajectory method in a study of 2,425 participants from the Washington Heights-Hamilton Heights-Inwood Community Aging Project (WHICAP) study, followed over 12 years with four visits, it was found that a higher initial level of depression symptoms predicted a steeper decline of memory both at the next visit, and over the entire follow-up study. Conversely, memory scores did not predict changes in depressive symptoms (Zahodne et al., 2014). In a conflicting study using an equivalent methodology, a bidirectional study of depressive symptoms and memory performance in $14,78950+$ years old participants from the Health and Retirement Study indicated that depressive symptoms did not predict change in memory. Instead, memory performance predicted depressive symptoms in a two-year later assessment (Jajodia \& Borders, 2011).

Despite the considerable strength of twin methodology, only a few longitudinal twin studies have sought to unravel cause and effect of the correlation between depressive symptoms and cognitive ability. Assessment of twin correlations and differences in follow-up studies can be used to investigate the sequential relationship between two traits and to deduce to what extent a phenotypic association between the two is caused by common underlying genetic mechanisms.
Using 1,237 male twins from the Vietnam Era Twin Study of Aging (VETSA), a low cognitive ability in early adulthood, but not cognitive change over the years was shown to correlate to development of depressive symptoms 35 years later $(r=0.16)$. The twin design further allowed the authors to conclude that this correlation was, to a large extent (77\%), due to shared genetics (Franz et al., 2011). The study did, however, only assess depressive symptoms at middle age and was thus not able to investigate the possibility of reverse causality. In addition, an earlier Swedish study included 146 monozygotic (MZ) and dizygotic (DZ) twin pairs discordant for dementia in a co-twin control study, thus controlling for shared early life conditions and (partially) for shared genetics. The study concluded that individuals with a history of depression were more likely to have dementia than their non-depressed co-twin, but did not explore the reverse association and did not estimate the effect of genetic and environmental factors on the association (Brommelhoff et al., 2009).

In the present study, we made use of a large longitudinal population-based sample of middle-aged Danish twins, comprising 2,866 twin individuals. This twin sample was recently used to assess the magnitude of genetic influences on individual differences in cognitive functioning over a 10-year interval. The results demonstrated that the genetic factors influencing cognitive functioning was nearly perfectly correlated over the two assessments, and that individual differences in change in cognitive functioning is overwhelmingly due to non-shared environmental factors (McGue et al., 2015). Here, we have applied similar twin modeling approaches extended to two phenotypes to shed light on the genetic correlation between both level and change of depression symptomatology and cognitive functioning, and to further explore the bidirectionality of any such correlation using assessments of both phenotypes at both occasions.

\section{Methods and Materials Participants}

The Study of Middle Aged Danish Twins (MADT) was initiated in 1998. The baseline sample consisted of 2,866 (1,267 pairs in which both members participated) same-sex twins (an additional sample of 1,448 twins from opposite-sex pairs were included in the survey but have been excluded in the present analyses). Twins were randomly drawn from all available twin pairs through the Danish Twin Registry, and evenly distributed across zygosity and birth cohorts 19311952. At an in-person, home-based visit, trained interviewers from the Danish National Institute of Social Research administered an extensive interview that included questions on mental and physical health as well as cognitive and physical tests (Skytthe et al., 2013). In 2008-2010, the surviving baseline twins were invited to participate in a 10 -year follow-up. The follow-up assessment included a self-report 
questionnaire filled out by the twins in advance of an interview by nurses or medical students who carried out the physical and mental evaluations at a hospital or, in case the participants did not live near a hospital, at home visits. A total of 1,582 (including 557 pairs in which both members participated) same-sex twins (55.2\% of all intake participants and $61.8 \%$ of surviving intake participants) took part in the follow-up assessment. Zygosity was established by means of the twins' responses to a short mailed questionnaire that included four questions on physical similarity and difficulty in separately identifying the two members of a pair by individuals who were close to the twins (Christiansen et al., 2003). At baseline, 1,459 (50.9\%) of the twins were classified as MZ and 1,407 (49.1\%) as same-sex DZ; at follow-up the numbers were $807 \mathrm{MZ}(51.0 \%)$ and $775 \mathrm{DZ}$ $(49.0 \%)$, respectively. Mean age was 56.8 years $(S D=6.4)$ at baseline and 66.5 (6.2) years at follow-up. The percentage of males was $51.3 \%$ at baseline and $54.0 \%$ at follow-up.

\section{Phenotypic Measures}

Cognitive composite score. A composite cognitive score was computed by aggregating the participants' performance on six subtests (forward and backward recall of digit sequences of length 3-9 and 2-8, respectively, immediate and delayed recall of 12 nouns, number of animals mentioned within 1 minute, and a timed symbol-digit replacement; McGue \& Christensen, 2002). Each of the six component scores (at baseline as well as at follow-up) was standardized using the means and standard deviations of the participants, who were less than 50 years of age at baseline. The cognitive composite was formed by adding these six standardized cognitive components. If one component was missing, the composite score was multiplied by $6 / 5$, and if more than one component was missing, the composite score was coded as missing. Subsequently, the composite score was $t$ score standardized (i.e., mean $=50$ and $S D=10$ ).

Affective depression score. Assessment of depressive symptomatology was performed using an adaptation of the depression section of the Cambridge Mental Disorders of the Elderly Examination (CAMDEX), which directly assesses both affective and somatic complaints (Johnson et al., 2002).

Only the affective depression subscale was used in the present study, as only these items were administered at both intake and follow-up. The affective depression assessment was based on nine questions about the respondents' current emotional state, with two questions rated on a 2-point scale, and the remaining seven questions rated on a 3-point scale. The affective score was the sum of the nine items arranged such that higher affective depression score reflects a higher level of depression. The affective depression score was not normally distributed, and moreover, the skewed distribution of the score was resistant to mathematical transforma- tions. Therefore, all the analyses were based on the original skewed scale.

\section{Statistical Methods}

Intrapair correlations. Structural equations modeling (SEM) was used to estimate intrapair correlations, for $\mathrm{MZ}$ and DZ twins separately, for affective and cognitive score at baseline and follow-up, as well as the change in the two scores between baseline and follow-up. Higher correlation in $\mathrm{MZ}$ than in DZ twins indicates that the etiology of the trait in question can partly be attributed to genetic factors. The cross-sectional correlations as well as the correlations of the 10-year change of the two phenotypes were estimated using SEM.

Biometric models. The phenotypic variance can be decomposed into a sum of four variance components: additive genetic factors (A), dominant genetic factors (D), shared environmental factors (C), and unshared environmental factors (E). Biometric modeling of twin data takes advantage of the difference of the genetic overlap of $M Z$ and DZ twins. While MZ twins share all of their genes, DZ twins share, as ordinary siblings, on average $50 \%$ of their genes. Therefore, the within-twin pair trait covariance of MZ twins can be expressed as cov (twin 1, twin2) $=\mathrm{A}+\mathrm{D}+\mathrm{C}$ and for DZ twins cov (twin 1, twin2 $)=0.5 \mathrm{~A}+0.25 \mathrm{D}+\mathrm{C}$. However, the full model is underspecified and it is therefore not possible to simultaneously identify the $\mathrm{C}$ and $\mathrm{D}$ components.

Using SEM, we fitted a bivariate two-factor growth model (Figure 1) to the data, whereby we simultaneously estimated the genetic and environmental contributions to the initial level and change of cognitive and affective depression scores (Neale \& Cardon, 1992). ACE and ADE models were fitted and it was tested whether $C$ and $D$, respectively, could be dropped without significant worse fit.

Heritability estimates of cognitive and affective scores at 10-year follow-up were separately estimated using univariate biometric AE-models.

To estimate the genetic and environmental contributions to the phenotypic (both within and between traits) correlations of the cognitive and affective scores at the two time points a bivariate SEM AE-models, using Cholesky decomposition, were fitted to pairwise combinations of affective and cognitive scores.

\section{Causality}

The analytical approach for establishing a possible causal link between affective depression and cognitive composite score followed three steps predicting causality. These have been described in detail by de Moor et al. (2008) and are briefly outlined in the following:

Prediction A (cross-sectional analysis). If trait A causes trait $\mathrm{B}$, it follows that environmental and genetic factors affecting trait A must also affect trait B. Thus, significant 


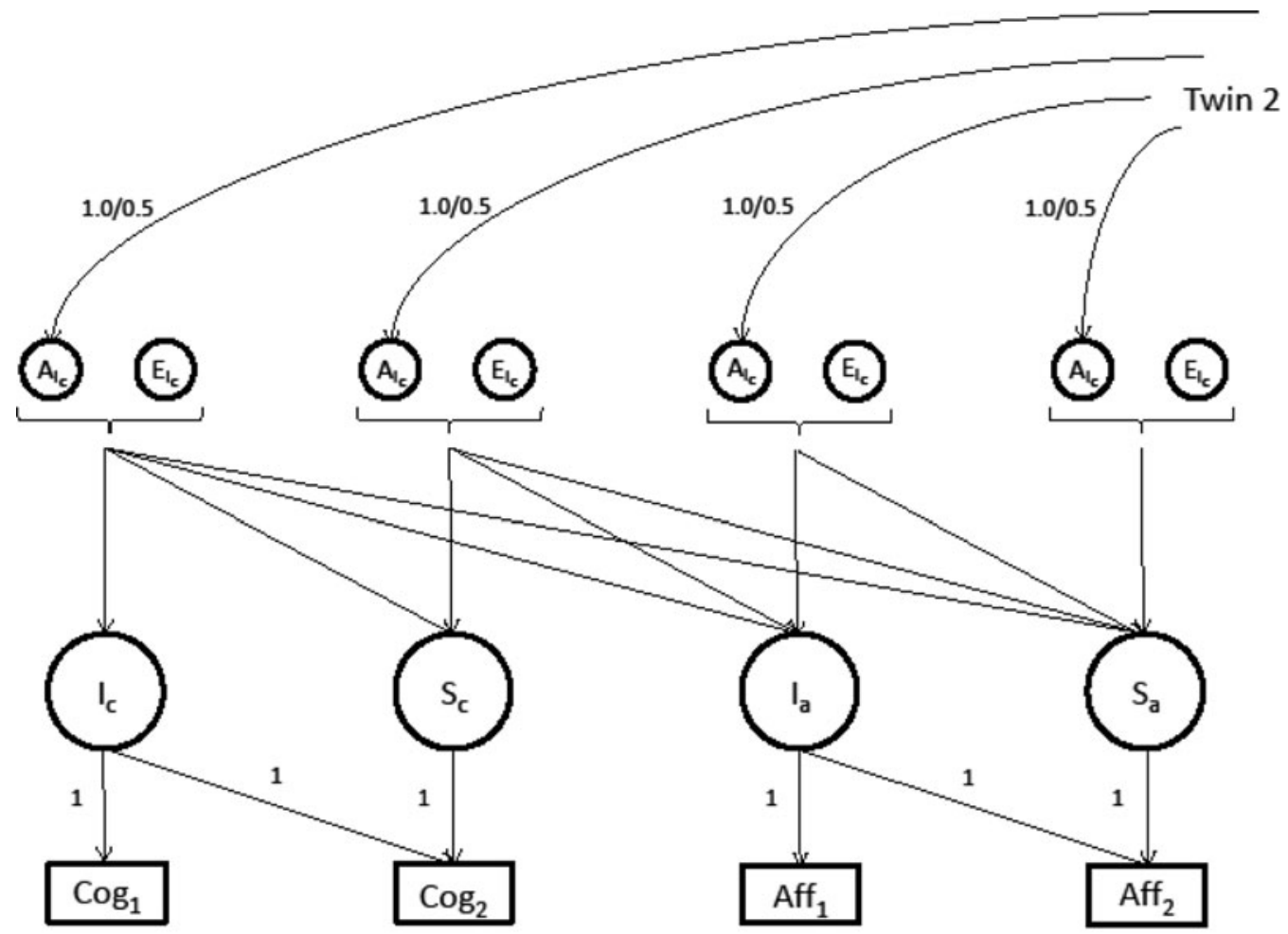

Twin 1

\section{FIGURE 1}

Path diagram for the 2-factor AE- growth model. $I_{c}$ and $I_{a}$ are the initial levels of cognitive and affective scores, respectively. $S_{c}$ and $S_{a}$ are the 10-year changes of the two scores.

environmental $\left(r_{\mathrm{e}}\right)$ and genetic correlations $\left(r_{\mathrm{a}}\right)$ are necessary conditions for a causal link between two phenotypes to exist. Bivariate structural equation modeling (SEM) was performed to examine the correlational structure of affective and cognitive scores at baseline as well as at follow-up. A second prediction formulated in de Moor et al.'s (2008) article claims that a causal link between two traits implies that the correlation between within-pair differences of the traits (co-twin control analysis) in MZ twins is significant. However, this requirement is comparable to the above requirement of $r_{\mathrm{e}}$ being significant.

Prediction B (longitudinal analysis). Second, a causal hypothesis requires temporal ordering (trait A precedes trait B) and so predicts that there should be significant environmental and genetic contributions to the correlation between baseline measures of trait A and follow-up measures of trait B. We used bivariate SEM to estimate the genetic and environmental contributions to the cross-trait crosstime correlations of affective score at baseline and cognitive score at follow-up and vice versa.

Prediction C (longitudinal analysis). A third analysis addressed the correlation of within-person changes for the two traits in question. A causal link between trait A and B would predict that within-person changes of trait A and B are correlated.

All analyses were conducted on residuals obtained from linearly regressing the cognitive scores and the affective score separately on age and sex. The Stata 13 software (StataCorp., 2013) was used for regression analyses and the $\mathrm{Mx}$ software (http://views.vcu.edu/mx/) was used for all analyses involving SEM analyses.

\section{Results}

The characteristics of the study sample as well as cognitive and affective scores are presented in Table 1, both for the entire sample and broken down by zygosity. The numbers demonstrate that it is the most cognitively intact and less depressed individuals at baseline who are most likely to participate at follow-up.

\section{Heritability}

Intrapair correlations for cognitive composite and affective scores at baseline, follow-up and 10-year change are reported in Table 2. The MZ correlation for the cognitive score was virtually unchanged from baseline to follow-up (0.61 and 0.64 , respectively) but the DZ correlation increased from 0.24 to 0.42 during the 10 years. Correlations 
TABLE 1

Sample Characteristics

\begin{tabular}{|c|c|c|c|c|c|c|}
\hline & \multicolumn{3}{|c|}{ Intake assessment } & \multicolumn{3}{|c|}{ Follow-up assessment } \\
\hline & All & $M Z$ & ssDZ & All & $\mathrm{MZ}$ & ssDZ \\
\hline Sample size & 2,866 & 1,459 & 1,396 & 1,582 & 807 & 775 \\
\hline Number of intact pairs & 1,267 & 664 & 603 & 557 & 308 & 249 \\
\hline Male percentage & $51.3 \%$ & $51.0 \%$ & $51.6 \%$ & $54.0 \%$ & $54.8 \%$ & $53.2 \%$ \\
\hline Mean $(S D)$ age & $56.8(6.4)$ & $56.9(6.4)$ & $56.8(6.3)$ & $66.5(6.2)$ & $66.5(6.1)$ & $66.5(6.3)$ \\
\hline Mean $(S D)$ cognitive score & $46.0(10.4)$ & $46.1(10.1)$ & $45.9(10.8)$ & $44.2(10.0)$ & $43.8(10.0)$ & $44.5(10.1)$ \\
\hline Mean $(S D)$ cognitive score - participants of both surveys & $48.1(10.0)$ & $48.0(9.8)$ & $48.1(10.4)$ & & & \\
\hline Mean $(S D)$ affective score & $10.7(2.4)$ & $10.6(2.4)$ & $10.8(2.4)$ & $10.9(2.3)$ & $10.9(2.3)$ & $11.0(2.4)$ \\
\hline Mean $(S D)$ affective score - participants of both surveys & $10.4(2.1)$ & $10.3(1.9)$ & $10.6(2.2)$ & & & \\
\hline
\end{tabular}

TABLE 2

Intrapair Correlations and Standardized Variance Components (AE Model) for Baseline Level, 10-Year Follow-Up and 10-Year Change of Age- and Sex-Adjusted Affective Depression and Cognitive Scores

\begin{tabular}{llllllll}
\hline & & \multicolumn{3}{c}{ Intrapair correlations } & & \multicolumn{2}{c}{ Variance components } \\
\cline { 3 - 4 } & & $\mathrm{MZ}$ & $\mathrm{DZ}$ & $\mathrm{p}\left(r_{\mathrm{MZ}}=r_{\mathrm{DZ}}\right)$ & & $\mathrm{a}^{2}[95 \% \mathrm{Cl}]$ & $\mathrm{e}^{2}[95 \% \mathrm{Cl}]$ \\
\hline Baseline & Cognitive & $0.61(0.56 ; 0.66)$ & $0.24(0.16 ; 0.31)$ & $<.001$ & & $0.62[0.57 ; 0.66]$ & $0.38[0.34 ; 0.43]$ \\
& Affective & $0.30(0.22 ; 0.37)$ & $0.12(0.04 ; 0.19)$ & .001 & & $0.28[0.21 ; 0.34]$ & $0.72[0.66 ; 0.79]$ \\
Follow-up & Cognitive & $0.64(0.57 ; 0.69)$ & $0.42(0.33 ; 0.51)$ & $<.001$ & & $0.65[0.59 ; 0.70]$ & $0.35[0.30 ; 0.41]$ \\
& Affective & $0.34(0.24 ; 0.44)$ & $0.19(0.08 ; 0.30)$ & $<.05$ & & $0.32[0.21 ; 0.41]$ & $0.68[0.29 ; 0.79]$ \\
10 -year change & Cognitive & $0.15(0.05 ; 0.26)$ & $0.05(-0.07 ; 0.17)$ & .20 & & $0.14[0.05 ; 0.23]$ & $0.86[0.77 ; 0.95]$ \\
& Affective & $0.04(-0.08 ; 0.16)$ & $0.01(-0.11 ; 0.13)$ & .73 & & $0.06[0 ; 0.17]$ & $0.94[0.83 ; 1]$ \\
\hline
\end{tabular}

for the affective scores increased slightly for $\mathrm{MZ}$ and $\mathrm{DZ}$ twins between the two time points.

In the two-factor growth models, $\mathrm{ACE}$ and $\mathrm{ADE}$ models were fitted to the data; however, the contribution from $\mathrm{C}$ $\left(\chi^{2}=5.59, d f=10, p=.85\right)$ and $\mathrm{D}\left(\chi^{2}=7.57, d f=10\right.$, $p=.67)$ did not reach statistical significance. Heritability estimates based on $\mathrm{AE}$ models revealed higher heritability for cognitive score ( 0.62 at baseline and 0.65 at follow-up) than for affective score ( 0.28 at baseline and 0.32 at followup) (Table 2). There was a small, but statistically significant, genetic contribution to the decline of cognitive score (0.14), but no genetic contribution to the change of the affective depression score.

\section{Causality}

Prediction A. These analyses demonstrated small but significant environmental and genetic contributions to the cross-trait correlation at baseline but only significant environmental contribution at 10-year follow-up (Table 3). Thus, the results at baseline are consistent with the hypothesis of a causal link between affective and cognitive scores. However, the analysis of cross-sectional data at follow-up did not confirm a significant genetic contribution to the cross-trait correlation and therefore failed to confirm the causal hypothesis.

Prediction B. Neither genetic nor environmental correlations demonstrated significance in bivariate longitudinal cross-trait analyses (Table 4). Thus, prediction B for causality is not fulfilled for either direction (i.e., affective depression score causing future cognitive score or vice versa).
Prediction C. The estimated correlation between 10-year change of affective depression and cognitive scores was small but significant (-0.08 [95\% CI: -0.13; -0.02]). Thus, withinperson change of one phenotype predicted parallel change of the other (e.g., increased affective depression score is associated with decreased cognitive score and vice versa). This result is consistent with a causal link between affective depression and cognitive scores.

\section{Discussion}

A considerable number of studies have over the years attempted to disentangle the correlation between the emergence of depressive symptoms and the onset of cognitive decline. Here, we used the powerful and genetically informative twin design, which allows for both estimation of the genetic contribution to cross-sectional and longitudinal cross-trait correlations, as well as for exploring any possible causal nature of such correlations.

Using 2,866 middle-aged twins, of whom 1,582 were assessed twice in 10 years, we found stable, occasion-specific (i.e., cross-sectional) heritability estimates of approximately $60 \%$ for general cognitive abilities and $30 \%$ for affective depressive symptoms. These estimates are completely consistent with our previous cross-sectional heritability estimates across age in Danish twin pairs (in which the present intake data was included; McGue \& Christensen, 2013; McGue et al., 2015), as well as with the heritability estimates from other studies (Carmelli et al., 2000; Jansson et al., 2004; Johnson et al., 2013). In addition, we saw a considerable decline in the mean cognitive performance over 10 years, 
TABLE 3

Cross-Sectional Phenotypic Correlation As Well As Genetic and Environmental Contribution to the Cross-Trait Correlation Estimated Through Bivariate SEM at Two Time-Points

\begin{tabular}{|c|c|c|c|c|c|}
\hline & \multicolumn{3}{|c|}{ Cross-trait correlations [95\% Cl] } & \multicolumn{2}{|c|}{$\begin{array}{l}\text { Percentage of phenotypic } \\
\text { correlation }\end{array}$} \\
\hline & Phenotypic & Genetic & Environmental & Genetic & Environmental \\
\hline Baseline & $-0.11(-0.14 ;-0.07)$ & $-0.14(-0.25 ;-0.02)$ & $-0.09(-0.16 ;-0.02)$ & $53.0 \%$ & $47.0 \%$ \\
\hline Follow-up & $-0.09(-0.14 ;-0.04)$ & $-0.01(-0.16 ; 0.14)$ & $-0.16(-0.27 ;-0.06)$ & $5.1 \%$ & $94.9 \%$ \\
\hline
\end{tabular}

Note: Statistically significant results $(p<.05)$ are shown in bold type.

TABLE 4

Phenotypic Correlation As Well As Genetic and Environmental Contribution to the Cross-Trait and Within-Trait Cross-Occasion Correlation Estimated Through Bivariate SEM

\begin{tabular}{|c|c|c|c|}
\hline & $r_{\mathrm{p}}[95 \% \mathrm{Cl}]$ & $r_{\mathrm{g}}[95 \% \mathrm{Cl}]$ & $r_{\mathrm{e}}([95 \% \mathrm{Cl}]$ \\
\hline Baseline affective and follow-up cognitive scores & $-0.07[-0.13 ;-0.01]$ & $-0.05[-0.21 ; 0.11]$ & $-0.10[-0.21 ; 0.01]$ \\
\hline Baseline cognitive and follow-up affective scores & $-0.02[-0.08 ; 0.03]$ & $-0.02[-0.17 ; 0.12]$ & $-0.03[-0.12 ; 0.07]$ \\
\hline Correlation of cognitive scores over 10 years & $0.69[0.66 ; 0.71]$ & $0.93[0.88 ; 0.98]$ & $0.25[0.16 ; 0.34]$ \\
\hline Correlation of affective scores over 10 years & $0.52[0.48 ; 0.56]$ & $0.92[0.76 ; 1.00]$ & $0.34[0.25 ; 0.42]$ \\
\hline
\end{tabular}

Note: $r_{\mathrm{p}}$ is the phenotypic correlation, $r_{\mathrm{g}}$ is the genotypic correlation, and $r_{\mathrm{e}}$ is the environmental correlation.

with a small, borderline significant genetic contribution $(14 \%)$. These results are overall in accordance with our recent detailed study of the genetic nature of cognitive level and change in these middle-aged twins, reaching the final conclusion that the change in cognition can be attributed mainly to non-shared environment (McGue et al., 2015). The minor genetic component seen in the present study is most likely due to the simpler modeling applied here, that is, we only included same sex twins, and we based our results on the AE model rather than the ACE model, to ease the simultaneous exploration of two phenotypes. Other equivalent studies have mainly been concerned with cognitive decline in individuals of higher age, where heritability estimates of change range from none to $18 \%$ (Lee et al., 2010). Contrary to our discovery here of a slight indication of a genetic contribution to cognitive change, we found the mean affective depression score to be stable over the 10-year follow-up period in middle-aged individuals, and with no indications of a genetic contribution to any individual change. While the temporal between-trait correlations were small, the stability correlations of the traits were 0.69 [95\% CI: $0.66 ; 0.71]$ for the cognitive score and 0.52 [ $95 \%$ CI: $0.48 ; 0.56$ ] for the affective score (Table 4 ). The temporal within-trait correlations were primarily due to common genetics - the genetic correlations were larger than 0.9 for both traits.

In accordance with previous studies, we did indeed see a small but significant and comparable cross-trait correlation at both occasions (-0.11 and -0.09 respectively). Surprisingly, the phenotypic correlation was influenced equally by genetics and environment at intake, but almost exclusively by environment at follow-up. This marked discrepancy in the genetic contribution may in part be caused by the fact that affective depression symptoms are variable, and highly impressionable to seasonal effects and occasion-specific circumstances such as recent adverse life events (McGue \& Christensen, 2003). In addition, it may be of importance that the participants are 10 years older at follow-up, and that only $55 \%$ participated in both assessments, although this hypothesis is contradicted by the fact that the mean depression level did not change over the years. In comparison, in the VETSA study of 1,237 male twins, Franz et al. (2011) found an $18 \%$ correlation between general cognitive ability and depression symptoms when the traits were assessed concurrently at middle age. They did not, however, estimate the overlapping genetic effect in their cross-sectional assessment (Franz et al., 2011). In any case, the correlations reported in the present study are generally small and only borderline significant, and thus we present only suggestive evidence of a shared genetic background for the two traits. Furthermore, from these estimates we cannot deduce if this indicates: (1) a genetic liability for depressed mood, which in turn impacts the cognitive abilities; (2) the reverse situation from this; or (3) a definite and genetically influenced pathological path, which is shared between the two phenotypic traits (Bennett \& Thomas, 2014).

Accordingly, a specific aim of this study was to contribute to the unraveling of the temporal relationship between cognitive decline and depression symptomatology, and to elucidate this question we followed the steps for causally linking two traits that were suggested by de Moor et al. (2008).

According to these criteria, a prerequisite for a causal association in one or the other direction would be a statistically significant influence from both genetics and the environment on the occasion-specific, cross-trait phenotypic correlation. Moreover, to infer a direction of such a causal association, the genetic contribution to any cross-trait longitudinal correlation should be significant in either one or 
the other direction, thus indicating that the one trait preceded the other. As we found only significant cross-trait genetic and environmental correlations in the intake assessment, the evidence for a causal relationship is weakened. However, the within-person change of one phenotype predicted parallel change of the other; for example, increased affective depression score was associated with decreased cognitive score and vice versa within the individuals, thus supporting a causal link between depression symptomatology and cognitive abilities. Finally, although the 10 year, longitudinal cross-trait analysis indicated a tendency towards depression symptoms acting as a predictor rather than a consequence of cognitive decline, all crosstrait, cross-occasion correlations were negligible. In total, our data thus provide no compelling evidence that either of the two traits predicts the onset of the other, over a 10-year interval. We cannot rule out, however, the possibility that the minor longitudinal correlations on top of the crosssectional correlations reflect the existence of shared genetic risk factors, which may promote an increase in affective depression symptoms at an earlier stage in life than the development of cognitive impairment.

Our results thus provide only very limited support to equivalent, but non-twin, studies with bidirectional longitudinal designs (Gale et al., 2012; van den Kommer et al., 2013; Zahodne et al., 2014), suggesting that depressive symptoms can predict future cognitive decline.

Despite the obvious advantage of twin studies in these research questions, this is the first twin study testing the bidirectional hypothesis. Two previous twin studies exist, and these are only partly in line with our results. In a combined case-control and discordant-twin study based on more than 12,000 participants in the Study of Dementia in Swedish Twins, Brommelhoff et al. (2009) found that those individuals who had a history of depression were more likely to have dementia than (unrelated) individuals with no history of depression. In support, a smaller sample of 146 twin $\mathrm{MZ}$ and $\mathrm{DZ}$ pairs discordant for dementia, of whom 40 had a history of depressive episodes, the co-twin control analysis revealed three times higher odds for the depressed twin to have dementia. Based on the latter study, the authors conclude that the association between dementia and preceding depression is not explained by genetics or shared early environment (Brommelhoff et al., 2009).

In their longitudinal analysis of VETSA, Franz et al. (2011) estimated a 16\% phenotypic correlation between general cognitive ability and depressive symptoms when assessed 35 years apart, whereas lack of intake data on depression prevented them from testing the reverse hypothesis. In striking contrast to our findings, they additionally attributed $77 \%$ of the correlation between young adulthood cognition and depression symptoms at middle age to shared genetics. Possible explanations for this discrepancy may be that the participants were noticeably younger at intake than those included in the present study, and the considerably longer follow-up time in VETSA (Franz et al., 2011).

Limitations to our study include the sizeable loss to follow-up, that is, only $55 \%$ of individuals participated in the second assessment. However, from Table 1 it is evident that the better performing participants are those most likely to participate at the 10-year follow-up survey, and thus the main effect of the loss to follow-up is probably a weakened power in our analysis. In addition, although we made use of a reasonably large study sample, this sample may nevertheless be insufficiently powered for detection of a predicting potency of either depression symptoms on cognitive decline or vice versa, which (as judged by the very minor correlations) may be rather subtle. A longer follow-up time than the 10 years employed here may be necessary for a larger effect. Finally, we have to stress that the CAMDEX depressive symptom score applied in this study is not well suited for classification into clinical depression of varying severity. Instead the affective score is more likely to represent the normal variation in affective mood. Similarly, the cognitive composite score does not necessarily constitute an instrument for early detection of an emerging dementia, but rather reflects the normative aging-related decline in cognitive functioning. It is thus possible that stronger correlations, and better predictive potential, will be more evident for categorically diagnosed major depression.

In conclusion, to our knowledge this is the first report of a bidirectional longitudinal study of the association between depressive symptomatology and cognition using a large and genetically informative twin sample. We found small but significant occasion-specific correlations between affective depressive symptoms and the level of general cognitive abilities, but we could not confirm any causal association between the two traits.

\section{Acknowledgments}

This work was supported by grants from The Lundbeck Foundation (R83-A8168) and The Danish National Program for Research Infrastructure (09-063256).

\section{References}

Barnes, D. E., \& Yaffe, K. (2011). The projected effect of risk factor reduction on Alzheimer's disease prevalence. Lancet Neurology, 10, 819-828.

Bennett, S., \& Thomas, A. J. (2014). Depression and dementia: Cause, consequence or coincidence? Maturitas, 79, 184190.

Brommelhoff, J. A., Gatz, M., Johansson, B., McArdle, J. J., Fratiglioni, L., \& Pedersen, N. L. (2009). Depression as a risk factor or prodromal feature for dementia? Findings in a population-based sample of Swedish twins. Psychology and Aging, 24, 373-384.

Byers, A. L., \& Yaffe, K. (2011). Depression and risk of developing dementia. Nature Reviews Neurology, 7, 323-331. 
Carmelli, D., Swan, G. E., Kelly-Hayes, M., Wolf, P. A., Reed, T., \& Miller, B. (2000). Longitudinal changes in the contribution of genetic and environmental influences to symptoms of depression in older male twins. Psychology and Aging, 15, 505-510.

Christiansen, L., Frederiksen, H., Schousboe, K., Skytthe, A., von Wurmb-Schwark, N., Christensen, K., ... Kyvik, K. (2003). Age- and sex-differences in the validity of questionnaire-based zygosity in twins. Twin Research, 6, 275-278.

da Silva, J., Goncalves-Pereira, M., Xavier, M., \& MukaetovaLadinska, E. B. (2013). Affective disorders and risk of developing dementia: Systematic review. British Journal of Psychiatry, 202, 177-186.

de Moor, M. H., Boomsma, D. I., Stubbe, J. H., Willemsen, G., \& de Geus, E. J. (2008). Testing causality in the association between regular exercise and symptoms of anxiety and depression. Archives of General Psychiatry, 65, 897-905.

Diniz, B. S., Butters, M. A., Albert, S. M., Dew, M. A., \& Reynolds, C. F. (2013). Late-life depression and risk of vascular dementia and Alzheimer's disease: Systematic review and meta-analysis of community-based cohort studies. British Journal of Psychiatry, 202, 329-335.

Franz, C. E., Lyons, M. J., O’Brien, R., Panizzon, M. S., Kim, K., Bhat, R., ... Kremen, W. S. (2011). A 35-year longitudinal assessment of cognition and midlife depression symptoms: The Vietnam era twin study of aging. American Journal of Geriatric Psychiatry, 19, 559-570.

Gale, C. R., Allerhand, M., \& Deary, I. J. (2012). Is there a bidirectional relationship between depressive symptoms and cognitive ability in older people? A prospective study using the english longitudinal study of ageing. Psychological Medicine, 42, 2057-2069.

Gao, Y., Huang, C., Zhao, K., Ma, L., Qiu, X., Zhang, L., ... Xiao, Q. (2013). Depression as a risk factor for dementia and mild cognitive impairment: A meta-analysis of longitudinal studies. International Journal of Geriatric Psychiatry, 28, 441-449.

Huang, C. Q., Wang, Z. R., Li, Y. H., Xie, Y. Z., \& Liu, Q. X. (2011). Cognitive function and risk for depression in old age: A meta-analysis of published literature. International Psychogeriatrics, 23, 516-525.

Jajodia, A., \& Borders, A. (2011). Memory predicts changes in depressive symptoms in older adults: A bidirectional longitudinal analysis. Journals of Gerontology Series B: Psychological Sciences and Social Sciences, 66, 571-581.

Jansson, M., Gatz, M., Berg, S., Johansson, B., Malmberg, B., McClearn, G. E., ... Pedersen, N. L. (2004). Gender differences in heritability of depressive symptoms in the elderly. Psychological Medicine, 34, 471-479.
Johnson, W., McGue, M., Gaist, D., Vaupel, J. W., \& Christensen, K. (2002). Frequency and heritability of depression symptomatology in the second half of life: evidence from Danish twins over 45. Psychol. Med., 32, 11751185.

Johnson, W., McGue, M., \& Deary, I. (2013). Normative cognitive aging. In D. Finkel \& C. A. Reynolds (Eds.), Behavioral genetics of cognition across the lifespan (pp. 135-167). New York: Springer.

Lee, T., Henry, J. D., Trollor, J. N., \& Sachdev, P. S. (2010). Genetic influences on cognitive functions in the elderly: A selective review of twin studies. Brain Research Reviews, 64, $1-13$.

McGue, M., \& Christensen, K. (2002). The heritability of level and rate-of-change in cognitive functioning in Danish twins aged 70 years and older. Experimental Aging Research, 28, 435-451.

McGue, M., \& Christensen, K. (2003). The heritability of depression symptoms in elderly Danish twins: Occasionspecific versus general effects. Behavior Genetics, 33, 83-93.

McGue, M., \& Christensen, K. (2013). Growing old but not growing apart: Twin similarity in the latter half of the lifespan. Behavior Genetics, 43, 1-12.

McGue, M., Pahlen, S., Skytthe, A., \& Christensen, K. (2015). Genetic and environmental contributions to cognitive change: A longitudinal study of Danish twins in mid- to late-life. Manuscript submitted for publication.

Neale, M., \& Cardon, L. (1992). Methodology of genetic studies of twins and families. Dordrecht: Kluwer Academic Publisher.

Skytthe, A., Christiansen, L., Kyvik, K. O., Bodker, F. L., Hvidberg, L., Petersen, I., ... Christensen, K. (2013). The danish twin registry: Linking surveys, national registers, and biological information. Twin Research and Human Genetics, 16, 104-111.

StataCorp. (2013). Stata Statistical Software: Release 13. College Station: TX: StataCorp LP.

van den Kommer, T. N., Comijs, H. C., Aartsen, M. J., Huisman, M., Deeg, D. J., \& Beekman, A. T. (2013). Depression and cognition: How do they interrelate in old age?. American Journal of Geriatric Psychiatry, 21, 398-410.

Vinkers, D. J., Gussekloo, J., Stek, M. L., Westendorp, R. G., \& van der Mast, R. C. (2004). Temporal relation between depression and cognitive impairment in old age: Prospective population based study. BMJ, 329, 881 .

Zahodne, L. B., Stern, Y., \& Manly, J. J. (2014). Depressive symptoms precede memory decline, but not vice versa, in non-demented older adults. Journal of the American Geriatrics Society, 62, 130-134. 